\title{
A COMPARISON OF VARIOUS NODAL DISCONTINUOUS GALERKIN METHODS FOR THE 3D EULER EQUATIONS
}

\author{
Michael Bergmann, Svetlana Drapkina, Graham Ashcroft, Christian Frey \\ German Aerospace Center (DLR), \\ Institute of Propulsion Technology, \\ Linder Höhe, 51147 Cologne, Germany \\ e-mail: \{Michael.Bergmann@dlr.de,Svetlana.Drapkina@dlr.de,Graham.Ashcroft@dlr.de, \\ Christian.Frey@dlr.de\}
}

Keywords: Discontinuous Galerkin, Collocation, Spectral Element Methods

\begin{abstract}
Recent research has indicated that collocation-type Discontinuous Galerkin Spectral Element Methods (DGSEM) represent a more efficient alternative to the standard modal or nodal DG approaches. In this paper, we compare two collocation-type nodal DGSEM and a standard nodal DG approach in the context of the three-dimensional Euler equations. The nodal $D G$ schemes for hexahedral elements are based on the polynomial interpolation of the unknown solution using tensor product Lagrange basis functions and the use of Gaussian quadrature for integration. In the standard nodal DG approach, we employ uniform interpolation nodes and Legendre-Gauss (LG) quadrature points. The two collocated DGSEM schemes arise from using either LG or Legendre-Gauss-Lobatto $(L G L)$ points as both interpolation and integration nodes. The resulting diagonal mass matrices and the ability to compute the fluxes directly from the solution nodes give rise to highly efficient schemes.

The results of the numerical convergence studies highlight, especially at high approximation orders, the performance improvement of the DGSEM schemes compared to the standard $D G$ scheme. Although having advantages in the evaluation of the boundary values over the LG-DGSEM, the lower degree of precision of the LGL quadrature negates this benefit. In addition, without the application of filtering techniques or over-integration, the lower integration accuracy of the LGL-DGSEM leads to numerical instabilities at stagnation points. Hence, the $L G-D G S E M$ is found to be the most efficient scheme as it is more accurate and robust for the considered test cases.
\end{abstract}




\section{INTRODUCTION}

Through the permanently growing standards of modern turbomachines with regard to emission and noise pollution, there is a great need for new and more efficient engine technologies. To accurately investigate such technologies, more efficient, flexible and precise numerical tools for the simulation of turbomachinery flows are essential.

In this regard, the discontinuous Galerkin (DG) finite element method has become a very popular method for the computation of unsteady flows as it combines an arbitrary order of accuracy with local data and algorithmic structures. In contrast to the standard finite element method, the polynomial interpolating functions are not restricted by continuity requirements between adjacent elements, cf. [12]. Due to the absence of global continuity, solutions at element interfaces are obtained by techniques similar to those used in upwind finite volume methods, leading to a stable finite element formulation even for advection-dominated problems. As a consequence of their combined robustness, flexibility and accuracy, DG methods are generally more computationally intensive and have higher storage requirements, cf. [1]. The necessity of high-order accurate representation of curved boundaries, as pointed out by [3], enhances both drawbacks.

As an efficient alternative to the standard nodal or modal approaches, spectral collocate forms of the DG space discretization on hexahedral elements have recently been developed, cf. [1, 2, 10, 16]. In these approximations, tensor product Lagrange polynomials are used to interpolate the solutions and Gaussian quadrature for the integrals. Applying the same points as interpolation and quadrature nodes, i.e. collocate them, gives rise to highly efficient schemes. Unfortunately, this choice limits the maximal polynomial degree, which can be exactly integrated. Therefore, numerical instabilities can occur as a consequence of the insufficient quadrature for handling nonlinearities of the governing equations and high-order geometries, cf [2,14].

In this paper, we compare three nodal DG schemes in the context of the three-dimensional Euler equations. In the first scheme, we employ uniform interpolation nodes and LegendreGauss (LG) quadrature points. As we don't exploit the collocation, this scheme serves as a reference nodal implementation. The two collocated DG methods arise from using LG or Legendre-Gauss-Lobatto (LGL) points as interpolation and integration nodes, respectively. No over-integration or modal-filtering will be used in any scheme. All DG schemes investigated have been integrated into DLR's in-house CFD code TRACE, which is developed at DLR's Institute of Propulsion Technology, cf. [9].

This paper is organized as follows: In Section 2, the governing equations and the DG methods investigated in this work are presented. In Section 3, the methods are then applied to two inviscid test cases. The last section summarizes the results.

\section{Discontinuous Galerkin Approximation}

\subsection{Governing equations}

Three-dimensional compressible inviscid flow is described by the Euler equations, which can be written in divergence form as

$$
\frac{\partial \boldsymbol{q}}{\partial t}+\nabla \cdot \boldsymbol{F}(\boldsymbol{q})=0
$$


with suitable initial and boundary conditions. The Cartesian components of the conservative state vector $\boldsymbol{q}$ and flux vectors $\langle\boldsymbol{F}(\boldsymbol{q}), \boldsymbol{n}\rangle=\boldsymbol{F}^{\boldsymbol{n}}(\boldsymbol{q})$ are

$$
\boldsymbol{q}=\left(\begin{array}{c}
\rho \\
\rho \boldsymbol{u} \\
\rho E
\end{array}\right), \quad \boldsymbol{F}^{\boldsymbol{n}}(\boldsymbol{q})=\left(\begin{array}{c}
\rho\langle\boldsymbol{u}, \boldsymbol{n}\rangle \\
\rho \boldsymbol{u}\langle\boldsymbol{u}, \boldsymbol{n}\rangle+p \boldsymbol{n} \\
\rho\langle\boldsymbol{u}, \boldsymbol{n}\rangle H
\end{array}\right)
$$

where $\langle\cdot, \cdot\rangle$ denotes the inner product, $\boldsymbol{n}=\left(n_{x}, n_{y}, n_{z}\right)$ is the outward normal vector to the boundary, $\rho$ is the density, $\boldsymbol{u}=(u, v, w)$ are the Cartesian velocity components, $E$ is the total energy and $H=E+p / \rho$ is the total enthalpy. The pressure is related to the other thermodynamic variables by the equation of state for an ideal gas and can be computed as $p=(\gamma-1) \rho\left(E-1 / 2\|\boldsymbol{u}\|^{2}\right)$, where $\gamma=1.4$ is the ratio between the specific heats of the fluid.

\subsection{Discontinuous Galerkin discretization}

The DG discretization of the Euler equations is based on the weak formulation, which can be obtained by multiplying Equation (1) by a sufficiently smooth test function $\boldsymbol{v}$ and performing integration by parts,

$$
\int_{\Omega}\left\langle\boldsymbol{v}, \frac{\partial \boldsymbol{q}}{\partial t}\right\rangle d \Omega+\int_{\partial \Omega}\left\langle\boldsymbol{v}, \boldsymbol{F}^{n}(\boldsymbol{q})\right\rangle d \sigma-\int_{\Omega} \nabla \boldsymbol{v}: \boldsymbol{F}(\boldsymbol{q}) d \Omega=0,
$$

where the symbol : denotes the double inner product. The domain $\Omega$ is now subdivided into shape-regular meshes $\mathcal{T}_{h}=\{K\}$ consisting of non-overlapping elements $K$, where $h$ denotes a piecewise constant mesh function. Hereafter, the solution $\boldsymbol{q}$ and test function $\boldsymbol{v}$ are approximated as piecewise polynomial functions $\boldsymbol{q}_{h}$ and $\boldsymbol{v}_{h}$, which are discontinuous across the elements. The space of piecewise polynomials is defined as,

$$
V_{h}=\left\{v_{h} \mid v_{h} \in L^{2}(\Omega), v_{h}\left(M^{K}(\boldsymbol{\xi})\right) \in \mathbb{P}^{p}\left(K_{\mathrm{ref}}\right), \forall K \in \mathcal{T}_{h}\right\},
$$

where the solution vector and the test function belong to $\boldsymbol{q}_{\boldsymbol{h}}, \boldsymbol{v}_{\boldsymbol{h}} \in\left(V_{h}\right)^{5}$. $\mathbb{P}^{p}\left(K_{\text {ref }}\right)$ denotes the space of tensor product polynomials up to degree $p$ on a reference element $K_{\text {ref }}$ and $M^{K}$ is a polynomial continuous invertible mapping for each element $K \in \mathcal{T}_{h}$ to a reference element $K_{\text {ref }}$ with $\boldsymbol{x}=M^{K}(\boldsymbol{\xi})$, where $\boldsymbol{x}=(x, y, z)$ are the Cartesian coordinates and $\boldsymbol{\xi}=(\xi, \eta, \zeta)$ the normalized coordinates of the reference element $K_{\text {ref }}$, cf. [3, 6, 9].

By substituting $\boldsymbol{q}$ with $\boldsymbol{q}_{h}$ and $\boldsymbol{v}$ with $\boldsymbol{v}_{h}$, the weak formulation (2) for each element $K \in \mathcal{T}_{h}$ can be rewritten as,

$$
\int_{K}\left\langle\boldsymbol{v}_{h}, \frac{\partial \boldsymbol{q}_{h}}{\partial t}\right\rangle d \boldsymbol{x}+\int_{\partial K}\left\langle\boldsymbol{v}_{h}, \mathcal{H}\left(\boldsymbol{q}_{h}^{+}, \boldsymbol{q}_{h}^{-}, \boldsymbol{n}\right)\right\rangle d s-\int_{K} \nabla_{h} \boldsymbol{v}_{h}: \boldsymbol{F}\left(\boldsymbol{q}_{h}\right) d \boldsymbol{x}=0 .
$$

Due to discontinuities of the solution $\boldsymbol{q}_{h}$ at element interfaces, the normal flux vector $\boldsymbol{F}^{\boldsymbol{n}}(\boldsymbol{q})$ in the boundary integrals is replaced by a normal numerical flux function $\mathcal{H}\left(\boldsymbol{q}_{h}^{+}, \boldsymbol{q}_{h}^{-}, \boldsymbol{n}\right)$, which depends on the outward normal vector $\boldsymbol{n}$, the internal interface state $\boldsymbol{q}_{h}^{-}$and the interface state of the neighboring element $\boldsymbol{q}_{h}^{+}$. In the current DG code the Roe numerical flux is employed, cf. [6]. 


\subsection{Polynomial approximation and numerical integration}

The approximated solution $\boldsymbol{q}_{h}$ and test function $\boldsymbol{v}_{h}$, belonging to the approximation space $\left(V_{h}\right)^{5}$, can be expressed as their polynomial expansion,

$$
\boldsymbol{q}_{h}(\boldsymbol{\xi}, t)=\sum_{i=1}^{N(p)} \boldsymbol{q}_{i}(t) \phi_{i}(\boldsymbol{\xi}), \quad \boldsymbol{v}_{h}(\boldsymbol{\xi})=\sum_{j=1}^{N(p)} \boldsymbol{v}_{j} \phi_{j}(\boldsymbol{\xi}), \quad \forall \boldsymbol{\xi} \in K_{\text {ref }} .
$$

Here, $\phi_{i}(\boldsymbol{\xi})$ denotes the basis function of the polynomial space $\mathbb{P}^{p}\left(K_{\text {ref }}\right), N(p)$ is the number of interpolation points, and $\boldsymbol{q}_{i}(t)$ and $\boldsymbol{v}_{j}$ are the expansion coefficients of $\boldsymbol{q}_{h}$ and $\boldsymbol{v}_{h}$, respectively. In this work, we consider nodal approximations and, therefore, using Lagrange interpolating polynomials as basis functions. In one space dimension, they are defined on the reference element as follows,

$$
l_{i}(\xi)=\prod_{j=1, j \neq i}^{p+1} \frac{\xi-\xi_{j}}{\xi_{i}-\xi_{j}}, \quad i=1, \ldots,(p+1),
$$

where $\xi_{j}$ are the node coordinates. One can easily observe that for Lagrange polynomials

$$
l_{i}\left(\xi_{j}\right)=\delta_{i j},
$$

holds, where $\delta$ is the Kronecker delta. For hexahedral elements, the nodal basis functions are the tensor product of one-dimensional Lagrange polynomials,

$$
\phi_{i}(\boldsymbol{\xi})=\phi_{m n o}(\xi, \eta, \zeta)=l_{m}(\xi) l_{n}(\eta) l_{o}(\zeta) .
$$

Considering the property of the Lagrange polynomials (7), the unknown expansion coefficients $\boldsymbol{q}_{i}(t)$ correspond to the solution values at the specific node $\boldsymbol{q}_{h}\left(\boldsymbol{\xi}_{i}, t\right)$, cf. [2].

Mapping Equation (4) into the reference space, substituting $\boldsymbol{q}_{h}$ by (5) and testing only against the basis functions $\phi_{j}(\boldsymbol{\xi})$ for all $j=1, \ldots, N(p)$, yields,

$$
\begin{aligned}
\frac{\partial \boldsymbol{q}_{i}(t)}{\partial t} \int_{K_{\mathrm{ref}}} \phi_{j} \phi_{i}\left|\boldsymbol{J}_{K}(\boldsymbol{\xi})\right| d \boldsymbol{\xi}+ & \int_{\partial K_{\mathrm{ref}}} \phi_{j} \mathcal{H}\left(\boldsymbol{q}_{h}^{+}, \boldsymbol{q}_{h}^{-}, \boldsymbol{n}\right) S_{K}(\boldsymbol{\xi}) d \hat{s}- \\
& \int_{K_{\mathrm{ref}}}\left\langle\left(\boldsymbol{J}_{K}(\boldsymbol{\xi})\right)^{-1} \nabla_{\xi} \phi_{j}, \boldsymbol{F}\left(\boldsymbol{q}_{h}\right)\right\rangle\left|\boldsymbol{J}_{K}(\boldsymbol{\xi})\right| d \boldsymbol{\xi}=0,
\end{aligned}
$$

where $\left|\boldsymbol{J}_{K}(\boldsymbol{\xi})\right|$ and $\left(\boldsymbol{J}_{K}(\boldsymbol{\xi})\right)^{-1}$ denotes the determinant and inverse of the Jacobian matrix, respectivly and $S_{K}(\boldsymbol{\xi})$ is the curved face area, cf. [9]. By assembling all element contributions, Equation (9) can be rewritten in the compact form as,

$$
\boldsymbol{M} \frac{\partial \boldsymbol{q}}{\partial t}+\boldsymbol{R}(\boldsymbol{q})=0
$$

Here, $\boldsymbol{M}$ denotes the mass matrix, $\boldsymbol{q}$ the unknown degrees of freedom (DOF) and $\boldsymbol{R}$ the residual vector, containing the boundary and volume integrals. The integrals are carried out using Gaussian quadrature formulae. For example, the mapped volume integral is approximated as,

$$
\begin{aligned}
\left.\int_{K_{\mathrm{ref}}}\left\langle\boldsymbol{J}_{K}^{-1}(\boldsymbol{\xi}) \nabla_{\xi} \phi_{j}(\boldsymbol{\xi}), \boldsymbol{F}\left(\boldsymbol{q}_{h}(\boldsymbol{\xi}, t)\right)\right\rangle \mid \boldsymbol{J}_{K}(\boldsymbol{\xi})\right) \mid d \boldsymbol{\xi} \approx \\
\sum_{i=1}^{M}\left\langle\boldsymbol{J}_{K}^{-1}\left(\boldsymbol{\xi}_{i}\right) \nabla_{\xi} \phi_{j}\left(\boldsymbol{\xi}_{\boldsymbol{i}}\right), \boldsymbol{F}\left(\boldsymbol{q}_{h}\left(\boldsymbol{\xi}_{\boldsymbol{i}}, t\right)\right)\right\rangle\left|\boldsymbol{J}_{K}\left(\boldsymbol{\xi}_{i}\right)\right| w_{i},
\end{aligned}
$$




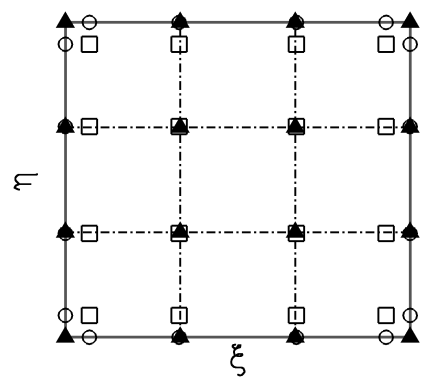

(a) DGNODAL

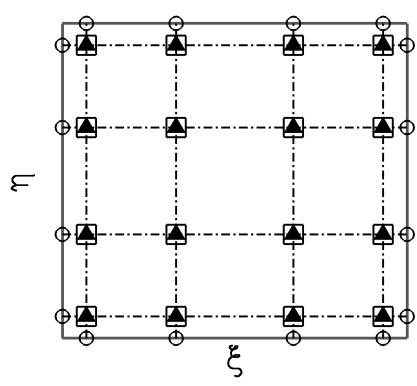

(b) LG-DGSEM

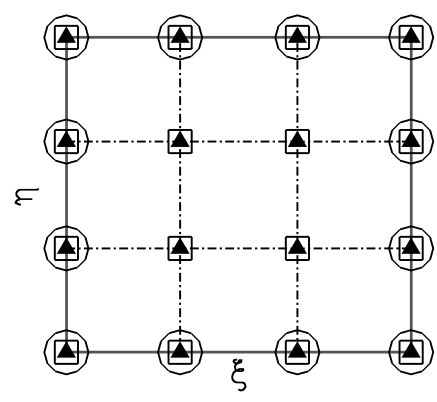

(c) LGL-DGSEM

Figure 1: Interpolation and quadrature nodes on the reference quadrilateral for an approximation of order $p=3$. The black triangles denote the interpolation nodes, the squares the volume quadrature points and the circles the boundary quadrature points of the specific scheme.

where $\boldsymbol{\xi}_{i}=\left(\xi_{i}, \eta_{i}, \zeta_{i}\right)$ and $w_{i}$ denotes the quadrature coordinates and weights, respectively. It is now possible to choose various sets of interpolation and quadrature nodes in order to derive different nodal DG formulations. Note, for all schemes presented, the number of quadrature points is set to be equal to the number of interpolation points, e.g. $M=N(p):=(p+1)^{3}$ for the three-dimensional case.

In the first scheme, we employ equidistant interpolation nodes and LG points as integration nodes. Note, although equidistant interpolation nodes can lead to unstable formulations for very high-order schemes, they are viable for moderate approximation orders, cf. [8, 11]. The degree of accuracy of the Gaussian quadrature with LG quadrature nodes is $2 M-1$, which means that polynomials up to degree $2 M-1$ are integrated exactly. In the following, this formulation is called DGNODAL.

For the purpose of efficiency, we consider nodal collocation-type approximations in the next schemes, i.e. using the same points as interpolation and integration nodes. As a result, many numerical operations can be omitted, which give rise to highly efficient implementation, cf. [2, 15, 16]. Two collocation formulations arise from using LG points or LGL points as both interpolation and quadrature nodes. With LG nodes, the degree of accuracy of the Gaussian quadrature is the same as in the DGNODAL formulation. Whereas, the degree of accuracy of the Gaussian quadrature with LGL nodes is only $2 M-3$. Hence, the inner product of two polynomial functions of order $p$, e.g. appearing in the mass matrix, is not integrated exactly, cf. [13, 16, 18]. The collocation formulations are named in the following as LG-DGSEM and LGL-DGSEM, respectively.

In order to clarify the DG schemes investigated in this work, the volume and boundary quadrature points and interpolation nodes for the two-dimensional case are shown in Figure 1 . The DGNODAL scheme represents the reference case in which all numerical operations are performed. Therefore, the solution and the derivatives have to be interpolated onto all the volume and boundary quadrature points. Additionally, the resulting element mass matrix is a dense matrix. Taking the interpolation nodes as integration nodes and considering the property of Lagrange polynomials (7), the mass matrix becomes diagonalized in both DGSEM schemes. Furthermore, the solution is directly available at the quadrature points of the volume integral and does not have to be interpolated. Through tensor product basis functions, the derivatives 


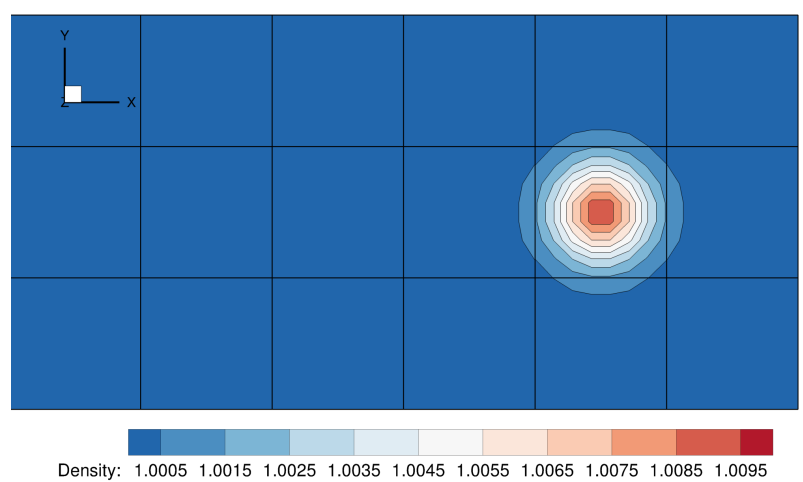

Figure 2: Gaussian pulse. Density contours of $\mathbb{P}^{7}$ solution on a coarse grid at $z=0.5$.

can be evaluated at the volume quadrature points using an one-dimensional interpolation. The disadvantage of the LG-DGSEM formulation, compared to the LGL-DGSEM, is however that boundary values have to be interpolated additionally, see Figures $1 \mathrm{~b}$ and $1 \mathrm{c}$.

\subsection{Explicit time integration}

An explicit three-stage third order accurate Runge-Kutta method is used to integrate Equation (10) in time. The scheme can be written as follows, cf. [6, 9],

$$
\begin{aligned}
\boldsymbol{q}^{(1)} & =\boldsymbol{q}^{n}+\Delta t \boldsymbol{R}_{M}\left(\boldsymbol{q}^{n}, t^{n}\right) \\
\boldsymbol{q}^{(2)} & =\frac{3}{4} \boldsymbol{q}^{n}+\frac{1}{4}\left(\boldsymbol{q}^{(1)}+\Delta t \boldsymbol{R}_{M}\left(\left(\boldsymbol{q}^{(1)}, t^{n}+\Delta t\right)\right)\right. \\
\boldsymbol{q}^{n+1} & =\frac{1}{3} \boldsymbol{q}^{n}+\frac{2}{3}\left(\boldsymbol{q}^{(2)}+\Delta t \boldsymbol{R}_{M}\left(\left(\boldsymbol{q}^{(2)}, t^{n}+\frac{1}{2} \Delta t\right)\right)\right.
\end{aligned}
$$

with $\boldsymbol{R}_{M}=\boldsymbol{M}^{-1} \boldsymbol{R}$. Applied to the DG system with elements of order $p$ this method has been shown to be linearly stable for a Courant number less than or equal to $\frac{1}{2 p+1}$, cf. [7].

\section{Numerical Results}

\subsection{Gaussian pulse in density}

In order to verify the formal order of accuracy of the DG schemes in practice, we consider a test case with an analytical solution. Therefore, a three-dimensional Gaussian density fluctuation is initialized in a cuboid domain with straight sided edges, cf. [17]. The pressure and velocity fields are uniform. The initial distribution is as follows,

$$
\boldsymbol{q}_{0}=\left(\begin{array}{c}
\rho_{0}+a \exp \left[-\ln \left(2 \frac{\left(\boldsymbol{x}-\boldsymbol{x}_{0}\right)^{2}}{\sigma^{2}}\right)\right] \\
\rho_{0} \boldsymbol{u}_{0} \\
\frac{p_{0}}{\gamma-1}+\frac{\rho_{0}\left\|\boldsymbol{u}_{0}\right\|^{2}}{2}
\end{array}\right),
$$

where $\boldsymbol{x}_{0}$ denotes the initial peak coordinates, $\sigma$ the width of the fluctuation and $a$ the amplitude. The analytical solution can obtained by moving the Gaussian pulse with the specified convective velocity $\boldsymbol{u}_{0}$. In Figure 2 , the density contours of the $\mathbb{P}^{7}$ solution are displayed for a coarse hexahedral mesh. The convergence rates of the schemes are investigated by performing computations on increasingly fine grids. To ensure that the finite temporal accuracy of the time 
integration method does not influence the results, a small constant time-step size has be used in all simulations. In Figures $3 \mathrm{a}, 3 \mathrm{~b}$ and $3 \mathrm{c}$, the $L^{2}$-errors of the outlined schemes are plotted against the characteristic mesh size $h$, i.e. the element edge length. The black dash-dot lines illustrate the formal convergence rate $\mathcal{O}\left(h^{p+1}\right)$ of the DG method. It can be seen clearly that the schemes achieve the expected order of convergence.

To compare the efficiency, the $L^{2}$-error is plotted against the run time in Figure $3 \mathrm{~d}$. The solid, dashed and dash-dot lines indicate the LG-DGSEM, the LGL-DGSEM and the DGNODAL implementations, respectively. It can generally be observed that the higher-order schemes outperform the lower-order ones beyond a certain accuracy level. Looking at the $\mathbb{P}^{3}$ results, the theoretical efficiency disadvantage of DGNODAL, compared to both DGSEM schemes, is also clearly evident. Furthermore, the results show that the LG-DGSEM is the most efficient scheme for the inspected approximation orders. Therefore, the higher accuracy of the Gaussian quadrature seems to offset the cost of the additional interpolation required at the element boundaries. However, as the efficiency advantage of the LG-DGSEM decreases with increasing approximation order, this implies that the benefit of the higher integration order decreases relative to the cost of the additional interpolation.

\subsection{Flow around a NACA0012 airfoil}

To investigate the behaviour of the schemes for flows with stagnation points and the usage of high-order boundaries, the inviscid flow around a NACA0012 airfoil is considered. The freestream Mach number is set to $M_{\infty}=0.3$ and an incidence angle equal to $\alpha=1.49^{\circ}$ is applied. The computations are performed on a coarse mesh containing 200 hexahedral elements with a single element in the spanwise direction. At the inlet and outlet boundaries Riemann boundary conditions are imposed. The profile geometry is represented by high-order elements of order $q=3$, which are generated using the process chain introduced in [9]. In Figure 4a, the Mach contours of the $\mathbb{P}^{3}$ solution of the LG-DGSEM scheme are presented. Same results can be obtained using the DGNODAL implementation. However, computations with LGLDGSEM turn out be unstable. These instabilities occur near the stagnation point of the leading edge. In [2], Bassi et. al. identified the numerical under-integration of the linear terms as the most critical reason for this phenomenon with the decoupling of the DOF at stagnation points being only the trigger of the numerical instabilities at lower orders. By using LGL interpolation and integration nodes with over-integration, i.e. $M=N+1$ and, therefore, applying noncollocation approximations, both possible reasons are nullified and a stable scheme for the test case can be obtained. Nevertheless, without collocation, the efficiency of the scheme is heavily impaired. Notice that this is just a brief test and has to be further investigated and optimized, cf. for example [4]. In Figure 4b, the pressure coefficient distributions on the profile of the $\mathbb{P}^{3}$ solutions of DGNODAL, LG-DGSEM and the over-integrated LGL scheme, respectively, are plotted. All schemes show very similar distributions.

In Figure 5a, the pressure coefficient distributions of various polynomial approximation orders, computed with LG-DGSEM, are plotted. The mesh and the geometrical approximation order is the same in all presented simulations, i.e. $q=3$. It can be observed that the distributions converge with increasing polynomial approximation order.

As a last observation, the pressure coefficient distribution of $\mathbb{P}^{5}$ LG-DGSEM is compared with solutions obtained with the second-order accurate finite volume (FV) solver in TRACE, cf. [5]. For this comparison two simulations have been performed with the FV solver: one on the coarse DG mesh with 200 elements and a second on a fine mesh with 9800 elements. The pressure coefficient distributions are illustrated in Figure $5 \mathrm{~b}$. As one can notice, there are 


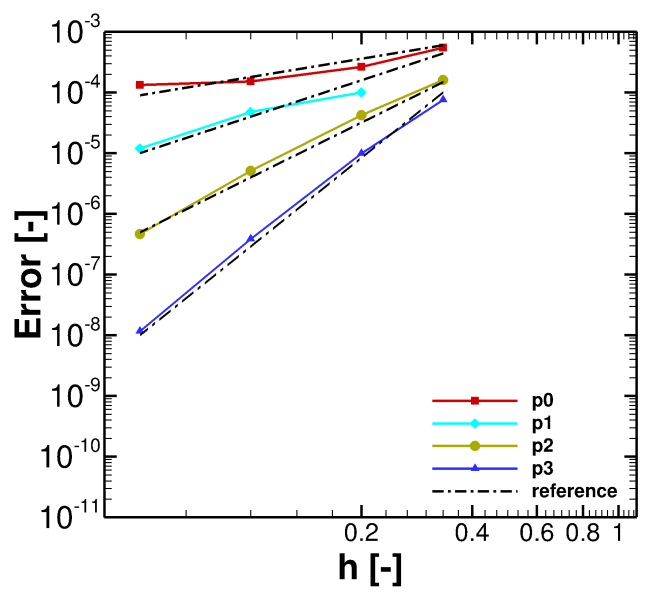

(a) DGNODAL

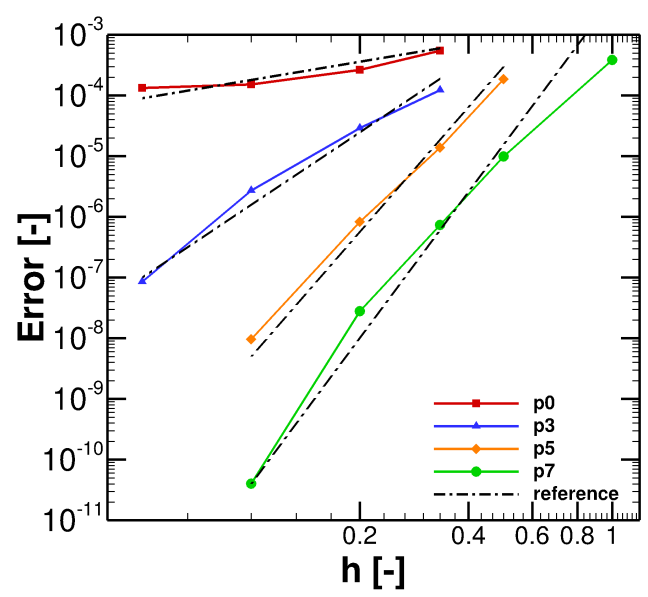

(c) LGL-DGSEM

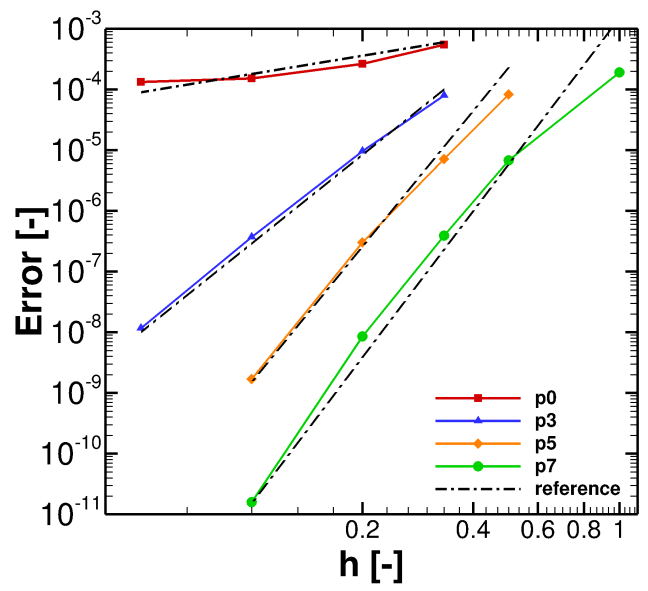

(b) LG-DGSEM

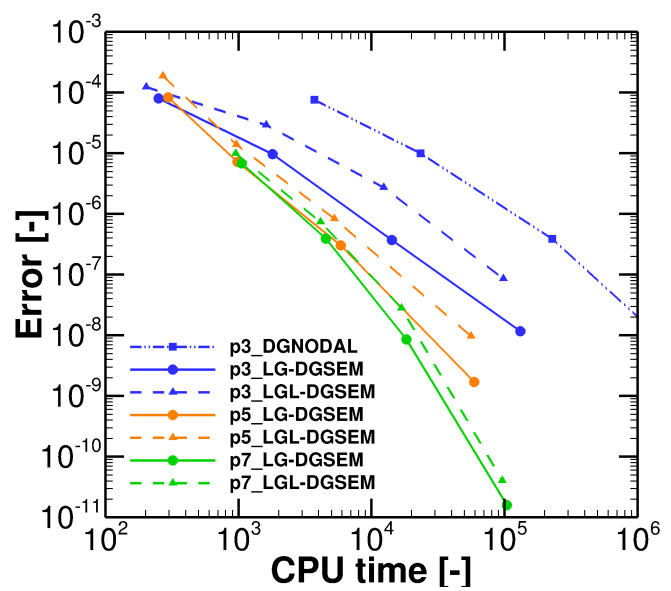

(d) CPU time behaviour

Figure 3: Gaussian pulse. Convergence rates and efficiency rates of the outlined schemes.

large differences between the FV solution on the coarse mesh and the other results. Moreover, the distribution of the fine FV simulation and $\mathbb{P}^{5}$ LG-DGSEM are very similar to each other. The slight differences are most likely due to the not fully converged mesh used for the fine FV simulation. However, these results suggest that the proposed DG scheme has the potential of computing highly accurate solutions.

\section{Conclusions}

In this paper, a standard nodal DG approach and two collocation-type nodal DG formulations are compared in the context of the compressible, three-dimensional Euler equations. All schemes considered show the optimal order of accuracy $\mathcal{O}\left(h^{p+1}\right)$ for an approximation order $\mathbb{P}^{p}$, whereby both collocation schemes have huge advantages in efficiency compared to the standard nodal DG approach. Matching both collocation methods, LG-DGSEM seems to be more efficient than LGL-DGSEM, although the difference between both schemes decreases with increasing polynomial approximation order as the influence of the lower integration order of LGL quadrature compared to the more efficient boundary value evaluation decreases. Furthermore, 


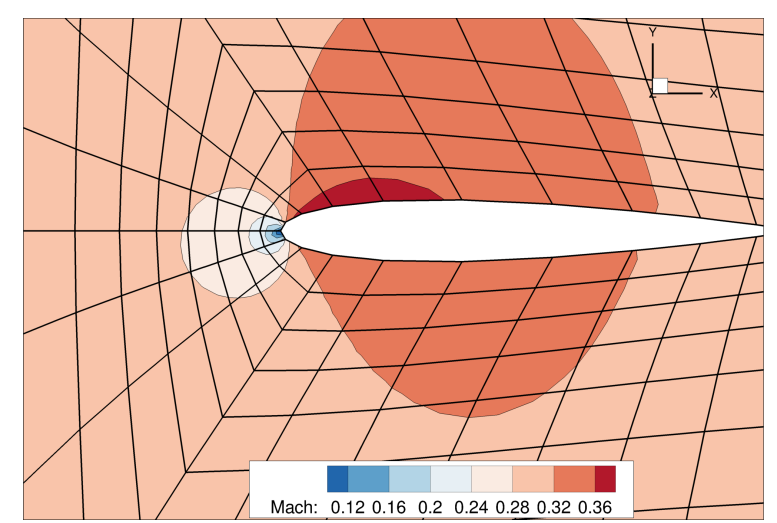

(a) LG-DGSEM

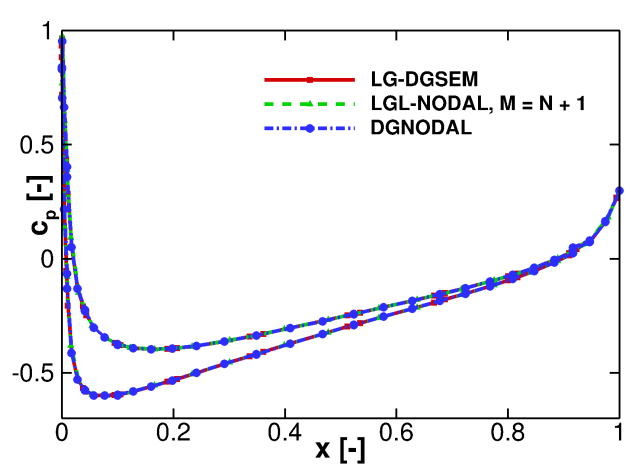

(b) Pressure coefficient distribution on the profile with $q=3$ of $\mathbb{P}^{3}$ solutions

Figure 4: NACA0012 airfoil, $M_{\infty}=0.3, \alpha=1.49^{\circ}$, geometric approximation of order $q=$ 3. Mach contours of the $\mathbb{P}^{3}$ solution with LG-DGSEM and pressure coefficient distributions obtained with LG-DGSEM, DGNODAL and the over-integrated, non-collocated nodal LGL formulation.

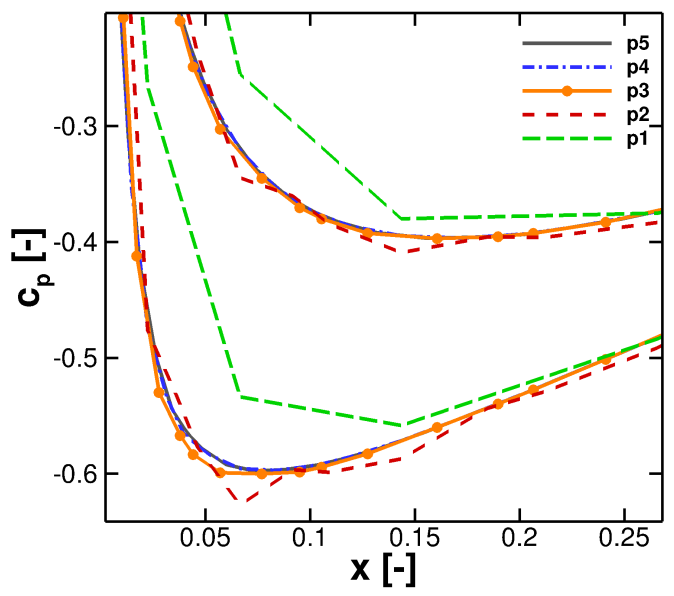

(a) LG-DGSEM, $p$-refinement, $q=3$

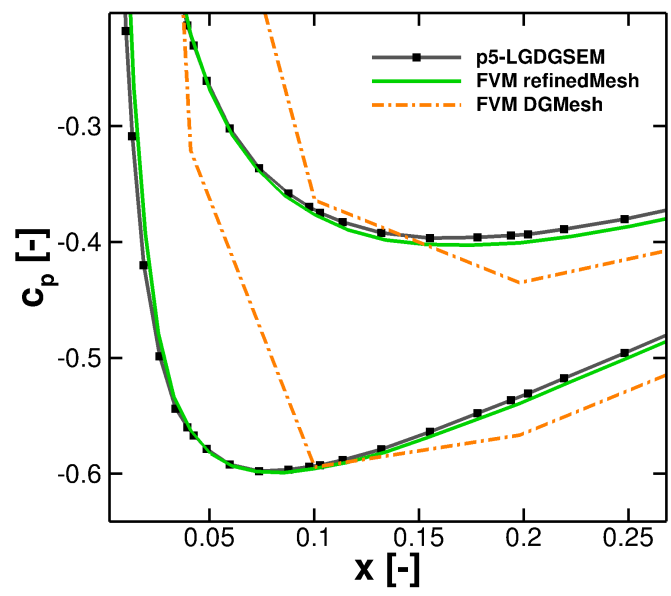

(b) $\mathbb{P}^{5}$ LG-DGSEM and 2nd order accurate FV solver

Figure 5: NACA0012 airfoil, $M_{\infty}=0.3, \alpha=1.49^{\circ}$. Pressure coefficient distributions. (a) LG-DGSEM with various polynomial approximation orders; (b) LG-DGSEM with $\mathbb{P}^{5}$ (solid black line with squares), 2nd order accurate FV scheme computed on the DG mesh, i.e. 200 hexahedral elements (orange dash-dot line) and on a refined mesh containing 9800 elements (green solid line), respectively.

the LGL-DGSEM, without applying over-integration or modal filter, becomes unstable at the stagnation point in the presented steady flow around a NACA0012 airfoil, whereas LG-DGSEM and DGNODAL remain stable. Hence, the LG-DGSEM is found to be the most efficient scheme as it is more accurate and robust for the considered test cases. Finally, comparisons with an extensively validated FV solver indicate the DG method is able to provide accurate results. 


\section{REFERENCES}

[1] F. Bassi, N. Franchina, A. Ghidoni, and S. Rebay. Spectral p-multigrid discontinuous Galerkin solution of the Navier-Stokes equations. International Journal for Numerical Methods in Fluids, 67(11):1540-1558, 2011.

[2] F. Bassi, N. Franchina, A. Ghidoni, and S. Rebay. A numerical investigation of a spectraltype nodal collocation discontinuous Galerkin approximation of the Euler and NavierStokes equations. International Journal for Numerical Methods in Fluids, 71(10):13221339, 2013.

[3] F. Bassi and S. Rebay. High-order accurate discontinuous solution of the 2D Euler equations. Journal of computational physics, 138:251-285, 1997.

[4] A. Beck. High order discontinuous Galerkin methods for the simulation of multiscale problems. PhD thesis, Faculty of Aerospace Engineering and Geodesy of the University of Stuttgart, 2015.

[5] K. Becker, K. Heitkamp, and E. Kügeler. Recent progress in a hybrid-grid CFD solver for turbomachinery flows. In Proceedings Fifth European Conference on Computational Fluid Dynamics ECCOMAS CFD 2010, 2010.

[6] S. Cherednichenko, C. Frey, and G. Ashcroft. On the application of the discontinuous Galerkin method to turbomachinery flows. In 6th European Congress on Computational Methods in Applied Sciences and Engineering (ECCOMAS 2012), pages 2359-2375, September 2012.

[7] B. Cockburn and C. Shu. Runge-Kutta discontinuous Galerkin methods for convectiondominated problems. Journal of Scientific Computing, 16(3):173-261, 2001.

[8] M. O. Deville, P. F. Fischer, and E. H. Mund. High-Order Methods for Incompressible Fluid Flow. Cambridge University Press, 2002.

[9] S. Drapkina, C. Frey, and G. Ashcroft. On the integration of high-order boundary elements in a 3d discontinuous Galerkin method for turbomachinery flows. In European Conference on Computational Fluid Dynamics - ECFD VI, Juli 2014.

[10] F. Giraldo, J. Hesthaven, and T. Warburton. Nodal high-order discontinuous Galerkin methods for the spherical shallow water equations. Journal of Computational Physics, 181(2):499 - 525, 2002.

[11] J. S. Hesthaven and T. Warburton. Nodal Discontinuous Galerkin Methods: Algorithms, Analysis, and Applications. Texts in applied mathematics. Springer, 2008.

[12] K. Hillewaert, N. Chevaugeon, P. Geuzaine, and J.-F. Remacle. Hierarchic multigrid iteration strategy for the discontinuous Galerkin solution of the steady Euler equations. International Journal for Numerical Methods in Fluids, 51(9-10):1157-1176, 2006.

[13] G. Karniadakis and S. Sherwin. Spectral/hp element methods for computational fluid dynamics. Oxford University Press, 2nd edition, 2005. 
[14] R. M. Kirby and G. E. Karniadakis. De-aliasing on non-uniform grids: algorithms and applications. Journal of Computational Physics, 191(1):249 - 264, 2003.

[15] D. A. Kopriva. Implementing Spectral Methods for Partial Differential Equations: Algorithms for Scientists and Engineers. Springer Publishing Company, Incorporated, 1st edition, 2009.

[16] D. A. Kopriva and G. Gassner. On the quadrature and weak form choices in collocation type discontinuous Galerkin spectral element methods. Journal of Scientific Computing, 44(2):136-155, 2010.

[17] B. Landmann. A parallel discontinuous Galerkin code for the Navier-Stokes and Reynoldsaveraged Navier-Stokes equations. PhD thesis, Faculty of Aerospace Engineering and Geodesy of the University of Stuttgart, 2009.

[18] S. A. Teukolsky. Short note on the mass matrix for Gauss-Lobatto grid points. Journal of Computational Physics, 283:408-413, 2015. 